\title{
Atividade respiratória e aspectos microbiológicos de cultivares de batatas minimamente processadas e armazenadas em diferentes temperaturas
}

\author{
Respiratory activity and microbiological aspects of minimally processed potatoes cultivars \\ stored at different temperatures
}

\author{
Maria Carolina Dario Vitti ${ }^{\mathrm{I}}$ Fabiana Fumi Sasaki ${ }^{\mathrm{I}}$ Patrícia Miguel ${ }^{\mathrm{II}}$ Angelo Pedro Jacomino ${ }^{\mathrm{III}}$ \\ Celso Luiz Moretti ${ }^{\text {IV }}$ Ricardo Alfredo Kluge ${ }^{I^{*}}$
}

\section{RESUMO}

O presente trabalho teve como objetivo avaliar aspectos fisiológicos e microbiológicos de diferentes cultivares de batatas minimamente processadas. Batatas das cultivares 'Ágata', 'Asterix' e 'Monalisa' foram selecionadas, classificadas, lavadas e descascadas por abrasão, sendo em seguida cortadas em cubos, sanitizadas e centrifugadas. Depois foram colocadas em frascos herméticos e armazenadas a 5, 15 e $25^{\circ} \mathrm{C}$. Foram determinadas a atividade respiratória e a produção de etileno logo após o processamento e durante 10 horas. As análises microbiológicas foram realizadas no dia do processamento e após nove, cinco e um dia de conservação, a 5,15 e $25^{\circ} \mathrm{C}$, respectivamente. Observou-se que as batatas 'Asterix' apresentaram maiores taxas respiratórias em relação às cultivares 'Ágata' $e$ 'Monalisa', independentemente da temperatura de armazenamento. Nas temperaturas de 15 e $25^{\circ} \mathrm{C}$, as batatas 'Ágata' e 'Monalisa' apresentaram comportamentos semelhantes entre si. As contagens de bactérias psicrotróficas e coliformes totais, para todas as cultivares armazenadas $5^{\circ} \mathrm{C}$, mantiveram-se dentro dos limites aceitáveis durante nove dias de armazenamento. Não foram detectados coliformes a $45^{\circ} \mathrm{C}$ e Salmonella. Ficou evidenciada a eficiência do armazenamento a $5^{\circ} \mathrm{C}$ no controle da atividade metabólica e na segurança microbiológica de batatas minimamente processadas.

Palavras-chave: Solanum tuberosum, taxa respiratória, microbiologia, processamento mínimo

\begin{abstract}
The purpose of the present research was to evaluate physiological and microbiological aspects of different minimally processed potatoes cultivars. Potatoes cultivar 'Agata', 'Asterix' and 'Monalisa' were selected, classified, washed and abrasion peeled before being diced, sanitized and centrifuged. They were then placed in hermetically closed flasks and stored at 5, 15 and $25^{\circ} \mathrm{C}$. The respiratory activity and ethylene production were determined immediately after the processing and during 10 hours. Microbiological analyses were carried out on the processing day and after one, five and nine days of storage at 25,15 and $5^{\circ} \mathrm{C}$, respectively. Potatoes cv. 'Asterix' showed the highest respiratory rates when compared to potatoes cultivar 'Agata' and 'Monalisa', regardless of the storage temperature. Potatoes cv. 'Agata' and 'Monalisa' behaved similarly to each other at 15 and $25^{\circ} \mathrm{C}$. Psychrotrophic bacteria and total coliforms counts for all cultivars were within acceptable limits at $5^{\circ} \mathrm{C}$ for nine days of storage. The presence of coliforms at $45^{\circ} \mathrm{C}$ and Salmonella was not detected. Storage at $5^{\circ} \mathrm{C}$ was effective to control the metabolic activity and to keep food safety of minimally processed potatoes.
\end{abstract}

Key words: Solanum tuberosum, respiratory rate, microbiology, minimal processing.

A qualidade de um produto minimamente processado é geralmente afetada por fatores précolheita e pós-colheita, incluindo o processamento. $\mathrm{O}$

\footnotetext{
IPrograma de Pós-graduação em Fisiologia e Bioquímica de Plantas, Escola Superior de Agricultura “Luiz de Queiroz” (ESALQ), Universidade de São Paulo (USP), Piracicaba, SP, Brasil.

IIDepartamento de Ciências Biológicas, ESALQ, USP. Av. Pádua Dias, 11, CP 9, 13418-900, Piracicaba, SP, Brasil. E-mail: rakluge@esalq.usp.br. *Autor para correspondência.

IIIDepartamento de Produção Vegetal, ESALQ, USP, Piracicaba, SP, Brasil.

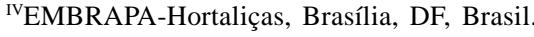


genótipo é um dos fatores mais importantes na précolheita, e o primeiro a ser avaliado a fim de selecionar cultivares a serem utilizados como produtos minimamente processados (CABEZAS-SERRANO et al., 2009). O frio tem importante papel na conservação de alimentos, pois a diminuição da temperatura retarda as transformações provocadas por reações bioquímicas e diminui a velocidade de crescimento de bactérias e fungos (ROLLE \& CHISM, 1987). O processamento mínimo tem sido uma das alternativas para agregar valor à batata produzida no Brasil, vindo a reduzir a importação do produto congelado. Considerando a importância econômica desse vegetal, existe uma lacuna na literatura consultada no que diz respeito à avaliação de cultivares nacionais com potencialidade de uso na forma minimamente processada (PINELI et al., 2006). Assim, o objetivo deste trabalho foi avaliar a atividade respiratória e produção de etileno, bem como alguns aspectos microbiológicos de três cultivares de batatas minimamente processadas.

As batatas das cultivares 'Ágata', 'Asterix' e 'Monalisa', foram colhidas na região de Itapetininga, São Paulo (SP), e levadas para o laboratório de Fisiologia e Bioquímica Pós-colheita do Departamento de Ciências Biológicas da Escola Superior de Agricultura "Luiz de Queiroz”, da Universidade de São Paulo (ESALQ/USP). Foram selecionados tubérculos firmes, com ausência de danos mecânicos e infecções. Com o objetivo de retirar as impurezas vindas do campo, as batatas foram pré-lavadas em água corrente.

O processamento mínimo foi adaptado de VITTI et al. (2004) e constou de: descascamento; prélavagem com água resfriada $\left(5^{\circ} \mathrm{C}\right)$ por dois minutos; corte em forma de cubos (1x1x1cm); sanitização em água clorada (200ppm de cloro ativo) por cinco minutos; centrifugação; pesagem (300g) e embalagem em filme plástico de nylon multicamadas a vácuo com dimensões $14 \times 20 \mathrm{~cm}$. As batatas minimamente processadas (MP) foram armazenadas nas temperaturas de $5 \pm 1^{\circ} \mathrm{C} ; 15 \pm 1^{\circ} \mathrm{C}$ e $25+1^{\circ} \mathrm{C}$ e $85 \%$ UR.

Para a avaliação da taxa respiratória, $150 \mathrm{~g}$ do produto MP foram colocados em frascos herméticos de vidro com septos de silicone nas tampas, que permaneceram fechados durante 30 minutos. Foi coletada uma alíquota de $1 \mathrm{~mL}$ de gás de cada frasco e injetada em cromatógrafo a gás (Thermoffinigan modelo GC Trace 2000) equipado com coluna Porapack N e detector de ionização de chama (FID), tendo hidrogênio como gás de arraste. As temperaturas foram de $80^{\circ} \mathrm{C}$ na coluna, $100^{\circ} \mathrm{C}$ no injetor, $250^{\circ} \mathrm{C}$ no detector e $350^{\circ} \mathrm{C}$ no metanador. As leituras foram feitas a cada hora, por 10 horas. Durante esse período, os frascos permaneceram nas temperaturas estudadas. Os resultados foram expressos em mL CO $\mathrm{kg}^{-1} \mathrm{~h}^{-1}$. Os procedimentos para determinação de etileno foram semelhantes aos utilizados para a determinação da taxa respiratória, porém os frascos permaneceram fechados por duas horas, e as temperaturas da coluna, do injetor e do detector do cromatógrafo foram de $80,100,100^{\circ} \mathrm{C}$, respectivamente. Os resultados foram expressos em $\mu \mathrm{L} \mathrm{C}_{2} \mathrm{H}_{4} \mathrm{~kg}^{-1} \mathrm{~h}^{-1}$.

As análises microbiológicas foram realizadas no dia do processamento e após nove, cinco e um dia de conservação a 5,15 e $25^{\circ} \mathrm{C}$, respectivamente. As análises para contagem de bactérias psicrotróficas foram realizadas por meio da técnica de plaqueamento em superfície (com meio PCA), enquanto que, para contagem de coliformes totais e coliformes a $45^{\circ} \mathrm{C}$, foi utilizada a Técnica de Tubos Múltiplos, ambas de acordo com VANDERZANT \& SPLITTSTOESSER (1992). Na análise da determinação da presença de Salmonella, foi empregado o kit rápido '1-2 test', fabricado pela BioControl/USA, conforme SILVA et al. (2001). Na avaliação dos aspectos microbiológicos, os resultados foram expressos em UFC/g de produto para bactérias psicrotróficas, $\mathrm{NMP} \mathrm{g}^{-1}$ para coliformes totais e fecais e presença/ausência de Salmonella em $25 \mathrm{~g}$ de produto. As análises do dia zero (dia do processamento) foram realizadas após três horas de armazenamento nas temperaturas de 5,15 e $25^{\circ} \mathrm{C}$.

O delineamento experimental foi inteiramente ao acaso. Para cada temperatura, foi utilizado esquema fatorial 3x10, cultivares (três níveis) e tempo de armazenamento (10 níveis). Foram utilizadas cinco repetições por tratamento. Os resultados das análises fisiológicas foram submetidos à análise da diferença mínima significativa em teste de comparações múltiplas, em que as diferenças das médias entre dois tratamento, maiores que a soma dos dois erros padrões, foram consideradas diferenças significativas a $5 \%$ de probabilidade (SHAMAILA et al., 1992).

Observou-se que a $5^{\circ} \mathrm{C}$ os valores da taxa respiratória foram mais baixos, independentemente da cultivar de batata (Figura 1). A cultivar 'Asterix' apresentou taxa respiratória significativamente $(\mathrm{P} \geq 0,05)$ mais elevada em todas as temperaturas, com valor médio de $16,25 \mathrm{~mL} \mathrm{CO}_{2} \mathrm{~kg}^{-1} \mathrm{~h}^{-1}$. A cultivar 'Monalisa' apresentou a menor taxa respiratória tendo e isso ficou bastante evidente na temperatura de $5^{\circ} \mathrm{C}$ (Figura $1 \mathrm{~A}$ ). A 15 e $25^{\circ} \mathrm{C}$ essa cultivar pouco diferiu da cultivar Ágata, principalmente nas primeiras quatro horas após o processamento.

Deve ser ressaltado que o estudo foi realizado até 10 horas após o processamento e que, após esse período, pode ocorrer menor atividade respiratória em razão da estabilização no metabolismo. Sabe-se que, quanto maior é a atividade respiratória de 


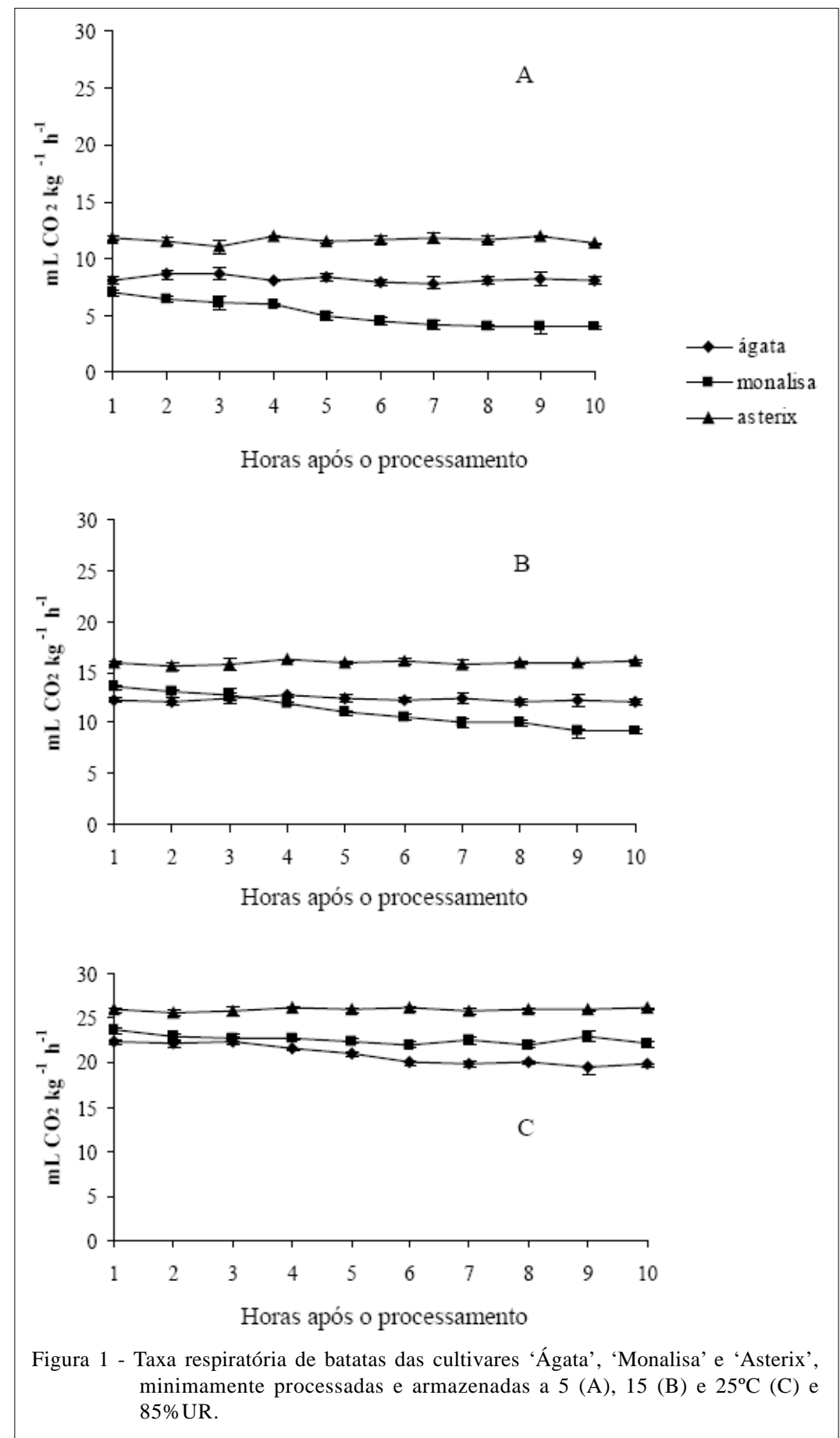

um vegetal, maior é o consumo de suas reservas energéticas, e menor é a sua vida de prateleira, devido ao consumo das reservas energéticas. Esse comportamento respiratório diferente entre as cultivares pode possivelmente ser em decorrência de diferenças existentes na quantidade de matéria seca presente nos diferentes genótipos. Assim, maiores teores de matéria seca podem significar maior fonte de substratos respiratórios.

De forma semelhante, MORETTI et al. (2002) observaram comportamento distinto na taxa respiratória de diferentes cultivares de batata-doce minimamente processadas e armazenadas a $3^{\circ} \mathrm{C}$. 
PINELI et al. (2004a), estudando a atividade de batatas 'Ágata' minimamente processadas, observaram que as batatas armazenadas a $15^{\circ} \mathrm{C}$ apresentam taxa de evolução de $\mathrm{CO}_{2} 66 \%$ maior do que as armazenadas a $5^{\circ} \mathrm{C}$. Em estudo feito com batatas da cultivar 'Asterix' minimamente processadas, ALMEIDA et al. (2004) constataram apenas um pequeno aumento na taxa respiratória de tubérculos armazenados a $15^{\circ} \mathrm{C}$ em relação aos armazenados a $5^{\circ} \mathrm{C}$. PINELI et al. (2004b), estudando a atividade de batatas da cultivar 'Monalisa' minimamente processadas, também verificaram uma redução significativa na taxa de evolução de $\mathrm{CO}_{2}$, passando de $6,44 \mathrm{~mL} \mathrm{CO}_{2} \mathrm{~kg}^{-1} \mathrm{~h}^{-1}$, após a primeira hora, para $3,45 \mathrm{~mL} \mathrm{CO}_{2} \mathrm{~kg}^{-1} \mathrm{~h}^{-1}$ ao final de quatro horas. O etileno não foi detectado em nenhuma das cultivares e temperaturas de armazenamento.

Como não existe uma legislação específica no país, para produtos minimamente processados, foram utlizados os parâmetros apresentados por MORTON (2001) para vegetais congelados e similares. O crescimento da população de bactérias psicrotróficas foi retardado pela redução da temperatura de armazenamento (Tabela 1). As batatas armazenadas a $5^{\circ} \mathrm{C}$, independentemente da cultivar, mantiveram a contagem total de bactérias psicrotróficas dentro do limite tolerado por MORTON (2001), chegando em torno de 5,0x104 UFC g-1 ${ }^{-1}$ para a cultivar 'Ágata', no 9o dia de

Tabela 1 - Contagem total de bactérias psicrotróficas em batatas das cultivares Ágata, Monalisa e Asterix minimamente processadas e armazenadas em diferentes temperaturas.

\begin{tabular}{|c|c|c|}
\hline \multirow{2}{*}{ Cultivares } & \multicolumn{2}{|c|}{-----------Dias após corte----------- } \\
\hline & $0^{*}$ & 9 \\
\hline 'Ágata' $5^{\circ} \mathrm{C}$ & $3,8 \times 10^{2}$ & $5,0 \times 10^{4}$ \\
\hline 'Monalisa' $5^{\circ} \mathrm{C}$ & $4,0 \times 10^{2}$ & $5,0 \times 10^{4}$ \\
\hline 'Asterix' $5^{\circ} \mathrm{C}$ & $4,1 \times 10^{2}$ & $5,2 \times 10^{4}$ \\
\hline \multirow{2}{*}{ Cultivares } & ---------Di & rte---------. \\
\hline & 0 & 5 \\
\hline ‘Ágata’ $15^{\circ} \mathrm{C}$ & $7,1 \times 10^{3}$ & $4,0 \times 10^{5}$ \\
\hline 'Monalisa' $15^{\circ} \mathrm{C}$ & $7,0 \times 10^{3}$ & $4,8 \times 10^{5}$ \\
\hline ‘Asterix’ $15^{\circ} \mathrm{C}$ & $7,2 \times 10^{3}$ & $4,6 \times 10^{5}$ \\
\hline \multirow{2}{*}{ Cultivares } & -------Di & rte-------- \\
\hline & 0 & 1 \\
\hline ‘Ágata’ $25^{\circ} \mathrm{C}$ & $4,5 \times 10^{6}$ & $7,2 \times 10^{7}$ \\
\hline 'Monalisa' $25^{\circ} \mathrm{C}$ & $4,9 \times 10^{6}$ & $7,9 \times 10^{7}$ \\
\hline 'Asterix’ $25^{\circ} \mathrm{C}$ & $4,7 \times 10^{6}$ & $7,2 \times 10^{7}$ \\
\hline
\end{tabular}

*Valores do dia 0 referem-se à análise realizada três horas após armazenamento nas respectivas temperaturas. armazenamento. Na temperatura de $15^{\circ} \mathrm{C}$, as batatas apresentaram contagem na escala de $10^{5} \mathrm{UFC} \mathrm{g}^{-1}$ já no 5o dia de armazenamento. Embora ainda esteja dentro de limites aceitos, a recomendação para consumo desse produto com tal contagem é perigosa, pois aumenta a probabilidade de desenvolvimento de microrganismos patogênicos. As batatas mantidas a $25^{\circ} \mathrm{C}$ apresentaram

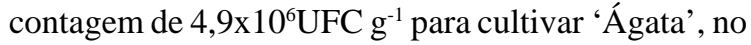
zero dia de armazenamento, e de $7,9 \times 10^{-7} \mathrm{UFC} \mathrm{g}^{-1}$ para variedade Monalisa no $\underline{1}^{\circ}$ dia de armazenamento, sendo esta muito elevada, o que torna o produto impróprio para o consumo, em razão dos riscos de contaminação.

IZUMI \& WATADA (1994) estudaram o crescimento microbiano em cenouras MP armazenadas nas temperaturas de 0,5 e $10^{\circ} \mathrm{C}$, durante 28 , 21 e 11 dias, respectivamente. Os autores notaram que a contagem microbiológica total foi maior para a temperatura mais alta. Após 15 dias, o produto estava com uma população de bactérias mesófilas totais de 7,0 log UFC g ${ }^{-1}$ a $0^{\circ} \mathrm{C}$ e acima de 8,01 log $\mathrm{UFC} \mathrm{g}^{-1}$ a $5^{\circ} \mathrm{C}$.

Embora não existam padrões para bactérias psicrotróficas totais e coliformes totais na legislação brasileira vigente, no que diz respeito à quantidade de microrganismos presentes em um alimento, pode-se afirmar que quantidades elevadas $\left(>10^{5} \mathrm{UFC} \mathrm{g}^{-1}\right)$ são indesejáveis, pelas seguintes razões: risco do alimento estar deteriorado, perda real ou potencial das qualidades organolépticas e comprometimento da aparência do alimento. Quanto maior o número de microrganismos em um alimento, maiores são as probabilidades de presença de patógenos e/ou deterioradores (CARUSO \& CAMARGO, 1984).

Os resultados obtidos nas análises microbiológicas estão dentro do limite estabelecido pela resolução RDC nำ12 de 02 de janeiro de 2001 da Agência Nacional de Vigilância Sanitária (ANVISA, 2007) do Ministério de Saúde. Pelas análises microbiológicas, principalmente a contagem de bactérias psicrotróficas, foi constatada a importância de se manter a cadeia de frio durante o processamento e a comercialização do produto, o que favorece uma maior conservação deste. Como não foram observados coliformes totais, coliformes a $45^{\circ} \mathrm{C}$ e Salmonella nas amostras analisadas em todos os tratamentos, ficou evidenciada a eficiência dos cuidados higiênico-sanitários tomados durante as etapas do processamento do produto, adequando-o aos padrões microbiológicos vigentes no país.

Existem efeitos do genótipo das cultivares nos processos fisiológicos, pois se observou que as batatas 'Asterix' apresentaram maiores taxas respiratórias em relação às cultivares 'Ágata' e 'Monalisa', independentemente da temperatura de 
armazenamento. A temperatura de armazenamento também tem efeitos nos processos fisiológicos e microbiológicos de batatas MP. Ficou comprovado que, quanto mais baixa a temperatura de armazenamento, menor é a taxa respiratória do produto. Além disso, o crescimento microbiano também foi reduzido com a redução da temperatura, permitindo a conservação das batatas MP por nove dias, a $5^{\circ} \mathrm{C}$.

\section{AGRADECIMENTOS}

À Fundação de Apoio à Pesquisa do Estado de São Paulo (FAPESP), pelo suporte financeiro (proc. n ${ }^{\circ}$ 2005/023324), à Coordenação de Aperfeiçoamento de Pessoal de Nível Superior (CAPES), pela concessão de bolsa de doutorado, e ao Conselho Nacional de Desenvolvimento científico e Tecnológico (CNPq), pela concessão de bolsa de produtividade em Pesquisa.

\section{REFERÊNCIAS}

ALMEIDA, G.C. et al. Comportamento fisiológico da batata cultivar asterix minimamente processada e intacta armazenada a 5 e $15^{\circ} \mathrm{C}$. In: ENCONTRO NACIONAL SOBRE PROCESSAMENTO MÍNIMO DE FRUTAS E HORTALIÇAS, 3., 2004, Viçosa, MG. Palestras... Viçosa: CEE, 2004. p.197.

ANVISA - AGÊNCIA NACIONAL DE VIGILÂNCIA SANITÁRIA. Resolução - RDC n 12, de 2 de janeiro de 2001. Capturado em 16 out. 2007. Online. Disponível em: <http:// e legis.anvisa.gov.br/leis ref/public/ showAct.php?id=144\&Word=limite\%20Microbiologico $>$.

CABEZAS-SERRANO, A.B. et al. Suitability of five different potato cultivars (Solanum tuberosum L.) to be processed as fresh-cut products. Postharvest Biology and Technology, v.53, p.138-144, 2009. Disponível em: <http://dx.doi.org/ doi:10.1016/j.postharvbio.2009.03.009>. Acesso em: 15 ago. 2009. doi:10.1016/j.postharvbio.2009.03.009.

CARUSO, J.G.B; CAMARGO, R. Microbiologia de alimentos. In: CAMARGO, R. (Ed.). Tecnologia dos produtos agropecuários-alimentos. São Paulo: Nobel, 1984. Cap.1, p.35-49.

IZUMI, H.; WATADA, A.E Calcium treatments affect storage quality of shredded carrots. Journal of Food Science, v.59, p.106-109, 1994.

MORETTI, C.L. et al. Respiratory activity and browing of minimally processed sweetpotatoes. Horticultura Brasileira, v.20, p.497-
500, 2002. Disponível em: <http://www.scielo.br/scielo.php? script $=$ sci_arttext\&pid $=$ S0102-05362002000300020\&lang $=p t>$. Acesso em: 01 out. 2008. doi: 10.1590/S0102-05362002000300020.

MORTON, R.D. Aerobic plate count. In: DOWNES, F.P.; ITO, K. Compendium of methods for the microbiological examinations of foods. Washington: American Public Health, 2001. p.63-67.

PINELI, L.L.O et al. Atividade respiratória de batatas ágata minimamente processadas armazenadas sob diferentes temperaturas. In: ENCONTRO NACIONAL SOBRE PROCESSAMENTO MÍNIMO DE FRUTAS E HORTALIÇAS, 3., 2004, Viçosa, MG. Palestras... Viçosa: CEE, 2004a. p.200.

PINELI, L.L.O et al. Atividade respiratória de batatas monalisa minimamente processadas e armazenadas em diferentes temperaturas. In: ENCONTRO NACIONAL SOBRE PROCESSAMENTO MÍNIMO DE FRUTAS E HORTALIÇAS, 3., 2004, Viçosa, MG. Palestras... Viçosa:CEE, 2004b. p.199.

PINELI, L.L.O et al. Caracterização química e física de batatas ágata e monalisa minimamente processadas. Ciência e Tecnologia de Alimentos, v.26, p.127-134, 2006. Disponível em: <http:// www.scielo.br/scielo.php?script=sci_arttext\&pid=S0101$20612006000100022 \&$ lang=pt $>$. Acesso em: 25 set. 2008. doi: 10.1590/S0101-20612006000100022.

ROLLE, R; CHISM, G.W. Phisiological consequences of minimally processed fruits and vegetables. Journal of Food Quality, v.10, p.157-165, 1987. Disponível em: <http:// www3.interscience.wiley.com/journal/119475671/abstract>. Acesso em: 03 ago. 2008. doi: 10.1111/j.17454557.1987.tb00856.x.

SHAMAILA, M. et al. Sensory evaluation of strawberry fruit stored under modified atmosphere packaging (MAP) by quantitative descriptive analysis. Journal of Food Science, v.57, p.1168-1172, 1992

SILVA, N. et al. Manual de métodos de análises microbiológicas de alimentos. São Paulo: Varela, 2001. 317 .

VANDERZANT, C.; SPLITTSTOESSER, D.F. Compendium of methods for the microbiological examination of foods. 3ed. Washington: APHA, 1992. 1219p.

VITTI, M.C.D. et al. Aspectos fisiológicos e microbiológicos de beterrabas minimamente processadas. Pesquisa Agropecuária Brasileira, v.39, p.1027-1032, 2004. Disponível em: <http:// www.scielo.br/ scielo.php?script=sci arttext\&pid=S0100204X2004001000011\&lang=PT>. Acesso em: 01 out. 2009. doi: 10.1590/S0100-204X2004001000011. 